\title{
Nitrate anion as a probe for electrostatic interactions in complexes protein-ligand
}

\author{
Smolina N. V. ${ }^{1}$, Dobretsov G. E. ${ }^{1}$, Syrejshchikova T. I. ${ }^{2}$, Gamburg Yu. D. ${ }^{3}$, Kalinina V. V. ${ }^{1}$, \\ Gryzunov Yu. A. ${ }^{1}$
}

${ }^{1}$ Research Institute for Physical Chemical Medicine, Moscow, Russia

${ }^{2}$ Lebedev Physical Institute, Moscow, Russia

${ }^{3}$ Frumkin Institute of Physical Chemistry and Electrochemistry, Moscow, Russia

\section{Email address:}

esmoline@mail.ru(Smolina N. V.), gdobretsov@newmail.ru(Dobretsov G. E.), syrej@sci.lebedev.ru(Syrejshchikova T. I.), varvara-bf@list.ru(Kalinina V. V.)

\section{To cite this article:}

Smolina N. V., Dobretsov G. E., Syrejshchikova T. I., Gamburg Yu. D., Kalinina V. V., Gryzunov Yu. A.. Nitrate Anion as a Probe for Electrostatic Interactions in Complexes Protein-Ligand. European Journal of Biophysics. Vol. 1, No. 2, 2013, pp. 22-27.

doi: 10.11648/j.ejb.20130102.12

\begin{abstract}
We are proposing a new technique for studying interaction of charged biomolecules. It is based on a difference between nitrate and chloride anion influence on electrostatic interactions in order to detect the role of positively charged guanidine groups of proteins. This comparison was demonstrated for human serum albumin (HSA) interaction with a fluorescent reporter, CAPIDAN. Nitrate anions lower their binding constant $(K)$. There are two causes that are responsible for that $K$ decrease induced by nitrate. The first, rise of the ionic strength (like to chloride), and the second, a specific one: at the same concentration and ionic strength nitrate anion more significantly lowers $K$ than chloride anion. The study of electric conductivity of chloride and nitrate salts shows that nitrate anions better than chloride anions form complexes with guanidine cations. Therefore it can be assumed that the nitrate-chloride techniques detect a direct contact of negatively charged carboxyl of CAPIDAN with positively charged arginine residues of HSA. HSA site I includes Arg 218 and $\operatorname{Arg} 222$. It is possible that the CAPIDAN carboxyl binds to one of these arginines.
\end{abstract}

Keywords: Human Serum Albumin, Drug-Binding Site, Ngatively Charged Ligands, Nitrate Anion Blocking Arginine Charge, Fluorescent Probe CAPIDAN

\section{Introduction}

Human serum albumin (HSA) transports many metabolites. To perform that function, special pockets (binding sites) exist in HSA molecules. Besides metabolites, these sites can bind xenobiotics including drugs as well as aromatic dyes $[1,2]$. Therefore such dyes, especially fluorescent ones, are widely used for the binding site study.

Recently one of the dyes, named CAPIDAN (Fig.1) [3], has been used to detect HSA molecule changes at some diseases, and some diagnostically important data have been obtained [4]. To clarify the nature of these changes in HSA binding sites, nitrate anions have been used: there is some data that nitrate is able to quench dynamically protein fluorescence and therefore it can be used for studying accessibility of protein fluorophores to water $[5,6]$.

We have tried to use the property of nitrate for testing HSA-bound CAPIDAN accessibility to water in pathologi- cal processes. Adding nitrate anion as well as chloride substitution by nitrate led to decrease of CAPIDAN fluorescence intensity $[7,8]$. However this effect is different in normal sera and at some psychic abnormalities $[7,8]$. This

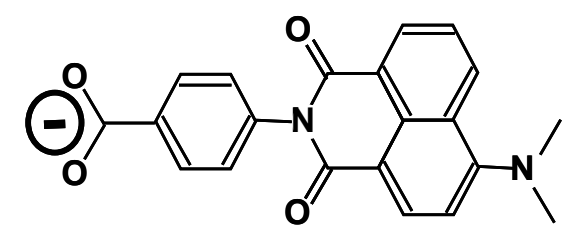

Figure 1. Fluorescent probe CAPIDAN, negatively charged at neutral pH.

study is an attempt to clarify mechanism of the fluorescence quenching by nitrate anions in the system fluorescent probe-HSA. Results that be obtained are found to be unexpected. 


\section{Methods}

Fluorescent probe CAPIDAN, N-(p-carboxyphenyl) imide of 4-(dimethylamino) naphthalic acid, has been synthesized and was kindly provided by B.M. Krasovitsky and colleagues (Institute of Monocrystalls, Kharkov, Ukraine).

Lyophilized powder of human serum albumin, fatty acid free, was purchased from Sigma-Aldrich (cat. A 1887). It was solved in a buffer, $0.01 \mathrm{~mol} /$ litre sodium phosphate +2 mmol/litre EDTA $\mathrm{pH}$ 7.4. This buffer was a base for all solutions used in fluorescence measurements. Guanidine hydrochloride and other salts were obtained from the same source.

Fluorescence spectra were measured using Hitachi F 4000 spectrofluorometer. Fluorescence decay was measured using a Pico-Quant instrument; the excitation source was a pulse diode with spectral maximum of $455 \mathrm{~nm}$; the Instrument Response Function, IRF, was close to 0.6 ns. CAPIDAN fluorescence decay was approximated as a sum of exponentials using the chi-squared criterion.

Conductivity of salt solutions was measured at $250 \mathrm{C}$ using conductometer InoLab Cond 720.

\section{Results}

CAPIDAN fluorescence intensity in water is very low, however it rises many times in the presence of HSA as a result of CAPIDAN binding to HSA [3, 4]. Increasing ionic strength leads to fluorescence decrease (Fig.2). Probably

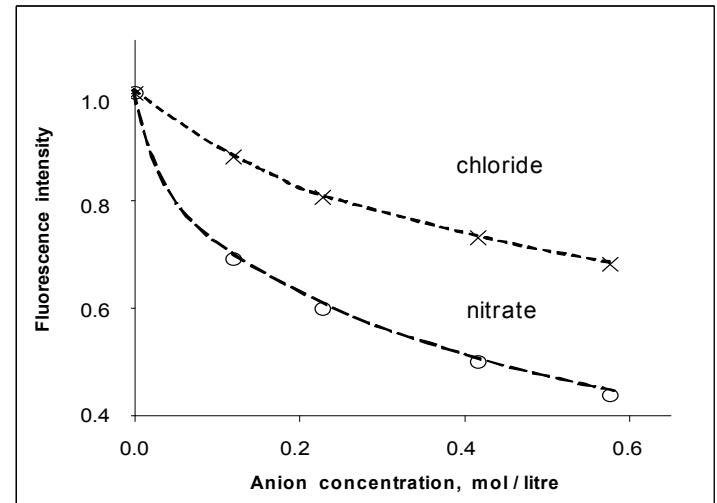

Figure 2. Lowering CAPIDAN fluorescence intensity in HSA solutions due to $\mathrm{KCl}$ or $\mathrm{KNO} 3$ addition. HSA $30 \mu \mathrm{mol} / \mathrm{litre}$, CAPIDAN $14 \mu \mathrm{mol} / \mathrm{litre}$.

the ionic strength rise suppresses Coulomb interaction of the CAPIDAN negative charge with positive charges of the HSA binding site [3] - like to other negatively charged aromatic ligands $[1,2]$ (some recent similar examples are $[9,10,11]$ etc. $)$.

Ionic strength rised by $\mathrm{NaCl}$ or $\mathrm{KCl}$ addition gives the same effect: i.e. cations $\mathrm{Na}+$ and $\mathrm{K}+$ are equivalent. Meanwhile nitrate anion suppresses the CAPIDAN fluorescence more effectively than chloride. So, two effects exist: an unspecific effect of the ionic strength rise and a specific action of nitrate on CAPIDAN fluorescence. That phenomenon is seen also in Fig. 3 where chloride anions were substituted equimolarly by nitrate at invariable ionic strength.

What is the cause of that difference between chloride and nitrate effects?

\subsection{A Hypothesis of the Dynamic Quenching}

Nitrate anions are able to quench dynamically the intrinsic protein fluorescence leading to decrease of fluorescence decay time $\tau$ [6]. Stern-Volmer description of a simple dynamic quenching [12] is

$$
1 / \tau=1 / \tau_{0}+\mathrm{k}_{\mathrm{q}}[\mathrm{Q}] ; \tau_{0} / \tau=1+\tau_{0} \mathrm{k}_{\mathrm{q}}[\mathrm{Q}]
$$

where $\tau_{0}$ and $\tau$ are the decay time in the absence and the presence of a quencher, respectively; $[Q]$ is the quencher concentration; $k_{q}$ is the rate constant of effective quencher-chromophore collisions. If the steady-state fluorescence intensity $F$ is proportional to $\tau$, then a common equation originates from (1) [13]:

$$
F_{0} / F=1+K_{S V}[Q]
$$

However the data of Fig. 2 cannot be described by the simple (2) (not shown). So, the existence of a simple dynamic quenching is not obvious. A more complex quenching can not be excluded on this step of the study because the initial CAPIDAN fluorescence decay in HSA has a complex nature $[3,4,14,15]$. Different CAPIDAN molecules in HSA have different environment producing three species of bound probe with three types of decay:

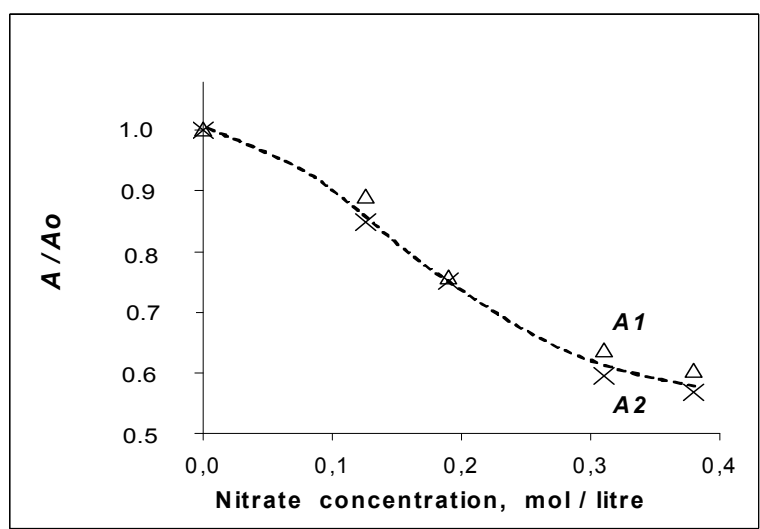

Figure 3. Lowering amplitudes of HSA-bound CAPIDAN fluorescence decay components due to equimolar substitution of chloride anions by nitrate. Summary concentration of chloride and nitrate was 0.63 mol/litre.AO is the amplitude without nitrate.

$$
F(t)=A_{1} \exp \left(-t / \tau_{1}\right)+A_{2} \exp \left(-t / \tau_{2}\right)+A_{3} \exp \left(-t / \tau_{3}\right)
$$

were $t$ is time after excitation. Amplitudes $A_{1}, A_{2}$ and $A_{3}$ are proportional to populations of these three species. $\tau_{1}$ value is close to $9.7 \mathrm{~ns}, \tau_{2}$ is near $3.6 \mathrm{~ns}$ and $\tau_{3}$ is about $1 \mathrm{~ns}$ (Table 1 ). Two first species are responsible for about 90-95\% (40-50 per cent each) of total fluorescence of HSA-bound CAPIDAN. Ionic strength rise does not influence decay times $\tau_{\mathrm{i}}$ and decreases all three amplitudes $A$; i.e. CAPIDAN binding to HSA decreases. 
Dynamic quenching should influence these $\tau_{\mathrm{i}}$. If nitrate dynamically quenches CAPIDAN fluorescence in HSA, then decay times should decrease in accordance with the Stern-Volmer theory (1). Meanwhile substitution of chloride by nitrate does not change any $\tau$ (Fig.4 and Table 1). So, the major sign of dynamic quenching, i.e. $\tau$ decrease, is absent. Nitrate lowers amplitudes $A_{\mathrm{i}}$. The most probable explanation of this phenomenon is suppressing CAPIDAN binding to HSA induced by nitrate. However nitrate does it significantly more effectively than chloride.

\subsection{Suppressing CAPIDAN-Albumin Binding Caused by Nitrate}

Nitrate influence on the CAPIDAN binding to HSA was tested using HSA titration by CAPIDAN. Data on $A_{1}$ changes are presented in Fig.5 as a double reciprocal graph proposed by Klotz [2]. $A_{1}$ is the amplitude of the first component of (3); it is proportional to number of the first species molecules bound to HSA and having $\tau_{1} \approx 9.7 \mathrm{~ns}$. Reciprocal value of CAPIDAN concentration is shown on horizontal axis. The experimental data can be fitted to a straight line. This line has an intercept on vertical axis equal to $1 / A_{1 \max }$ where $A_{1 \mathrm{max}}$ is proportional to number of HSA binding sites for the first CAPIDAN species. The intercept on the horizontal axis is approximately equal to the binding constant $\mathrm{K} 1$ for the species. As it was shown, even partial substitution of chloride by nitrate decreases $K_{1}$ by 2.9 times. In the case of CAPIDAN second species $\left(\tau_{2} \approx 3.6 \mathrm{~ns}\right) K_{2}$ decreases by 1.8 times (not shown). That substitution chloride/nitrate does not influence significantly number of binding sites (vertical intercepts); this is also a sign of absence of statically quenched CAPIDAN molecules.

Table 1. Parameters of CAPIDAN fluorescence decay in HSA solution as a function of the ionic strength at $\mathrm{NaCl}$ concentration rise.Amplitudes Ai are divided by their magnitudes at the lowest ionic strength $0.02 \mathrm{~mol} / \mathrm{litre}$. Decay time $\tau$ i error is $\pm 0.1 \mathrm{~ns}$.

\begin{tabular}{llll}
\hline Ionic strength, $\mathbf{~ m o l} / \mathbf{L}$ & $\mathbf{0 . 0 2}$ & $\mathbf{0 . 1 6}$ & $\mathbf{0 . 5 2}$ \\
\hline$A_{1} / A_{1}(0.02)$ & 1.00 & 0.52 & 0.32 \\
$A_{2} / A_{2}(0.02)$ & 1.00 & 0.63 & 0.44 \\
$A_{3} / A_{3}(0.02)$ & 1.00 & 0.80 & 0.62 \\
$\tau_{1}, \mathrm{~ns}$ & 9.7 & 9.7 & 9.8 \\
$\tau_{2}, \mathrm{~ns}$ & 3.9 & 3.6 & 3.6 \\
$\tau_{3}, \mathrm{~ns}$ & 1.1 & 1.0 & 1.0 \\
\hline
\end{tabular}

These data shows that chloride and nitrate anions suppress CAPIDAN binding to HSA but nitrate is significantly more effective at the same concentration. Nitrate lowers the binding constant $\mathrm{K}$ and does not influence number of binding sites.

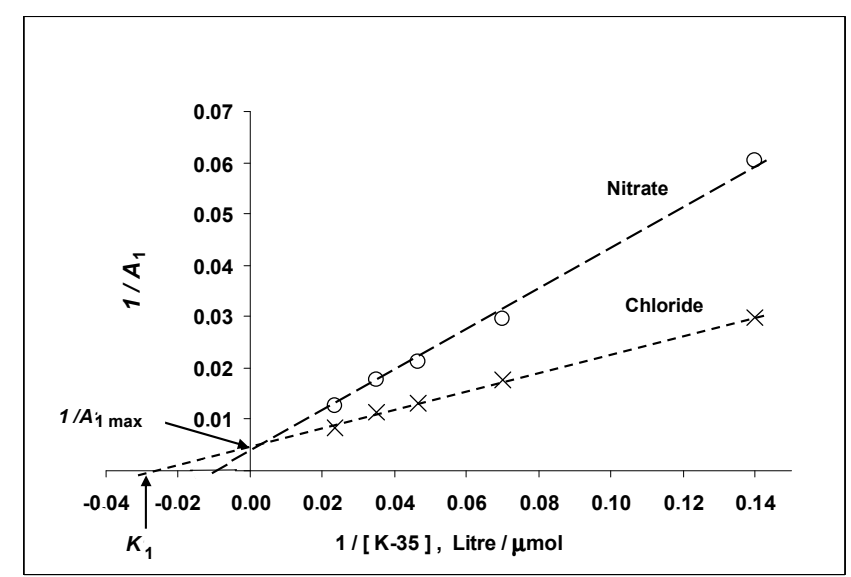

Figure 5. Titration of HSA by CAPIDAN in the presence of chloride 0.63 mol/litre and after equimolar substitution of $0.38 \mathrm{~mol} /$ litre chloride by nitrate. $A_{1}$ is amplitude of the CAPIDAN first species, $A_{\text {lmax }}$ is its maximal value. $K_{1}$ is the binding constant.

\subsection{Some Physical Chemical Differences Between Chlo- Ride and Nitrate Anions}

Why is nitrate a more effective suppressor of CAPIDAN binding than chloride? CAPIDAN binds with the first drug-binding site $[3,4,14]$. Negative charge of ligands promotes their binding to HSA $[1,2,10,11]$. Crystallographic data have shown $[16,17,18]$ that a cluster of positively charged lysine and arginine residues is localized on the boundary site I/water (Fig.6). Probably CAPIDAN negative charge interacts with this cluster; this hypothesis can explain the ionic strength effect (Fig.2 and Table 1) but does not clarify nitrate/chloride difference.

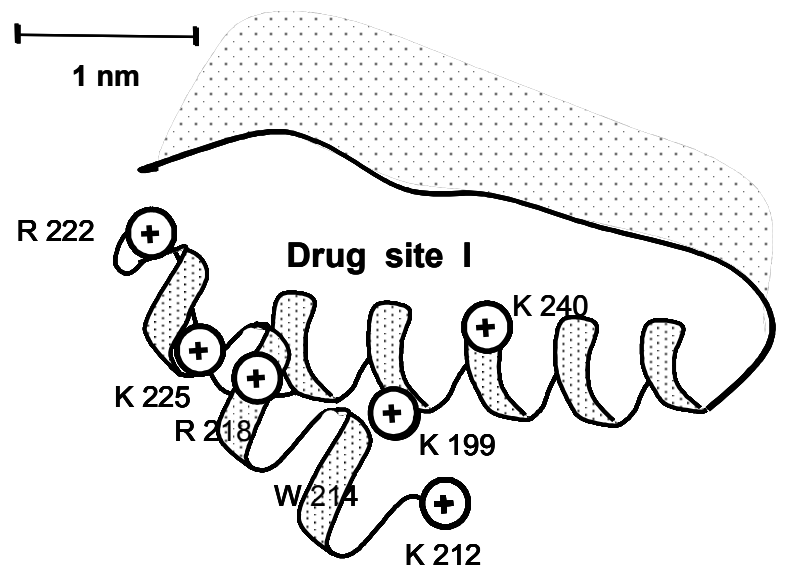

Figure 6. Schematic representation of entrance into the albumin first drug-binding site on the base of crystallographic data $[16 ; 17 ; 18]$. The single tryptophan residue $(W)$ and positively charged residues of lysine $(K)$ and arginine $(R)$ are shown.

It can be supposed that nitrate interacts with these HSA charges more strongly than chloride. May be, that interaction occurs with lysine ammonium groups, as it have been proposed by Klotz [1]. Indeed, as is known, there exists a significant difference between interaction of nitrate and 
chloride with ammonium: e.g. solubility of ammonium nitrate in water is 3.4 times more than ammonium chloride [19]. However in the case of CAPIDAN binding there is an inverse situation. At the same time solubility of guanidine nitrate is 6 times lower than guanidine chloride $[19,20]$. Thus, arginine guanidine groups could be assumed as probable targets for nitrate anions.

To obtain proof for this hypothesis, electric conductivity of guanidine salt solutions have been measured.

\subsection{Conductivity of Guanidine Salt Solutions}

Electric conductivity of salt solutions originates from ionic components of these solutions. So, $\mathrm{KCl}$ dissociates on $\mathrm{K}+$ and $\mathrm{Cl}-$ in the range of concentrations used above (see data of Fig.2-5 and Table 1). Conductivity of $\mathrm{KCl}$ solutions rises as a linear function of $\mathrm{KCl}$ concentration (Fig.7). This is a known fact [19]. Conductivity of guanidine chloride $(\mathrm{GuCl})$ is approximately the same as $\mathrm{KCl}$ (Fig.7), i.e. $\mathrm{GuCl}$ almost fully dissociates on $\mathrm{Gu}+$ and $\mathrm{Cl}-$, and moreover cations $\mathrm{Gu}+$ have conductivity close to $\mathrm{K}+$. Nitrate anions have conductivity close to chloride anions if the cation is $\mathrm{K}+$ [19]. Therefore in mixtures $\mathrm{K}+, \mathrm{Gu}+, \mathrm{Cl}-$ and $\mathrm{NO} 3-$ the total conductivity might be expected to be proportional to the total salt concentration $\mathrm{C}$. Results of conductivity changes due to $\mathrm{KCl}$ or $\mathrm{KNO} 3$ addition to $0.4 \mathrm{~mol} /$ litre guanidine hydrochloride are presented in Fig. 8 where conductivity is normalized on the total salt concentration. $\mathrm{C}$ here is close to ones in experiments on CAPIDAN binding to HSA (see above). As can be seen, there is a significant difference between $\mathrm{KCl}$ and $\mathrm{KNO} 3$ effects. In mixtures $\mathrm{K}+$, $\mathrm{Gu}+$ and $\mathrm{Cl}-$ this proportionality between conductivity and $\mathrm{C}$. is confirmed, and the ratio $/ \mathrm{C}$ is only very slightly dependent on $\mathrm{C}$ (Fig.8, upper curve). However it is not so in the case of mixing $\mathrm{Gu}^{+}$with NO3- where the ratio $/ \mathrm{C}$ falls

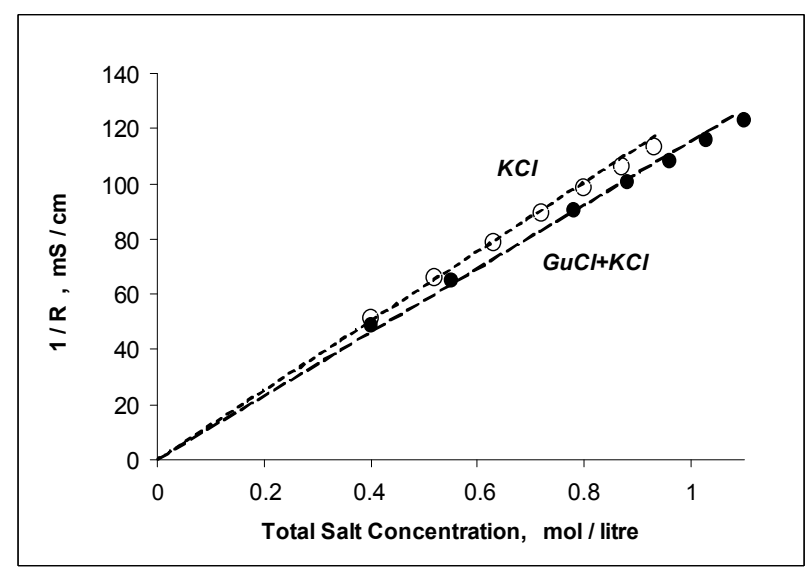

Figure 7. Conductivity of potassium and guanidine hydrochloride solutions. $\mathrm{KCl}$ (upper curve) or $\mathrm{GuCl}$ (lower curve) were added to $0.4 \mathrm{~mol} / \mathrm{L} \mathrm{KCl}$ solution. $R$-electric resistance.

significantly with $\mathrm{C}$ increasing (Fig.8, lower curve). The single explanation of that decrease is nitrate-guanidine complex formation because complexes anion-cation do not participate in conductivity.

Thus, conductivity data show that an interaction of nitrate anions with guanidine cations is stronger then chloride interaction with guanidine. As a result, nitrate shields the guanidine positive charge.

\section{Discussion}

Results presented above show that chloride and nitrate anions suppress binding of negatively charged fluorescent probe CAPIDAN with drug-binding site I of HSA but nitrate suppresses it more effectively. Obviously the binding strengthen due to Coulomb interaction of CAPIDAN charged carboxyl with positive charges of HSA [3]. Those charges can be attributed to amino groups of lysine or guanidine groups of arginine residues. Therefore, the rise of the ionic strength by chloride or nitrate leads to lowering CAPIDAN binding. At the same time, nitrate has an additional, specific effect. Conductivity data show that nitrate anions form uncharged complexes with guanidine cations while chloride with the same concentration almost does not. This fact allows to suggest that nitrate specific effect on CAPIDAN binding is due to shielding arginine charges. There is data on CAPIDAN binding with HSA drug site I [4,14]. In this site of crystallized HSA, a cluster of positive charges including Arg218 and Arg222 exists [16,17,18]. Data of neutron [21] and X-ray small-angle scattering [22] show that size of HSA molecule is similar in crystals and solutions; perhaps, the site I structure is similar as well. So, it can be suggested that negatively charged carboxyl of CAPIDAN binds with Arg218 or Arg222 of the site I, and nitrate suppresses this interaction. Binding of an anionic drug, warfarin, to the same site significantly enhances after Lys199 replacement by an uncharged residue but falls threefold after Arg218 replacement [16,23]. These

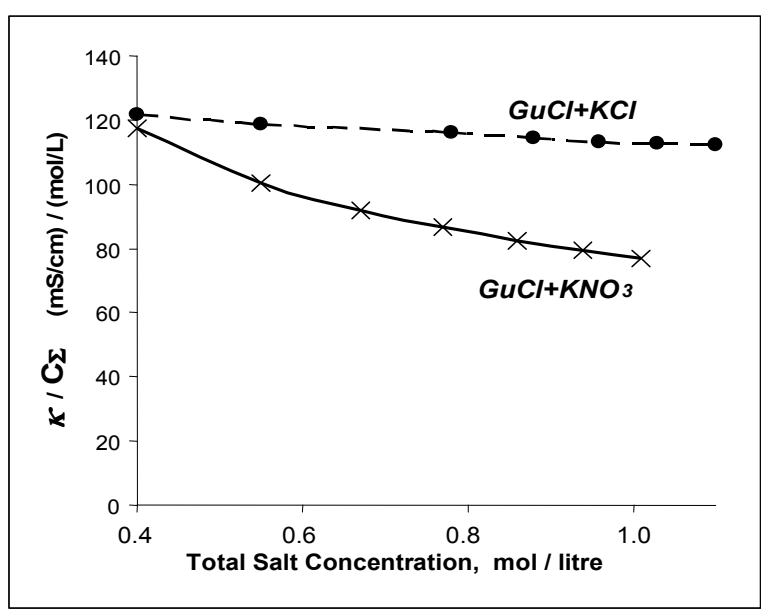

Figure 8. Ratio of the mean molar conductivity . and the total salt concentration $C_{\Sigma}$ of 0.4 mol/litre $\mathrm{GuCl}$ water solution after addition $\mathrm{KCl}$ (upper curve) or KNO3 (lower curve).

data support the suggestion that CAPIDAN also interacts with arginines of the site I and nitrate anions hinder this 
interaction.

\section{Conclusions}

Electrostatic interactions of charged amino acid residues play a very important role in formation of complexes protein-protein as well as protein-hormone, drug etc. Meanwhile contribution of different residues into these interactions is unknown very often. The data proposed above shows a way to detect participation of the arginine charge in those interactions. This approach has been tested on complexes of human serum albumin (HSA) with a fluorescent probe as a ligand. This complex is formed due to different forces including electrostatic [3]. Comparing the effects of nitrate and chloride anions on the interaction allows to detect the contribution of arginine residues. So, nitrate anion can be assumed to be a novel probe to study interaction of charged ligands with arginine positive charges of HSA and, probably, other proteins in solutions.

That interaction is significant if the distance between CAPIDAN carboxyl and this HSA guanidine group is rather small, i.e. several Angström. Therefore some small conformational changes of the protein binding site, including pathology-induced, could be detected using nitrate as a probe.

\section{Acknowledgments}

This work was partially supported by the International Science and Technology Center (ISTC Project \#3156), and the Russian Academy of Sciences program "Basic Sciences - to Medicine".

\section{References}

[1] I.M. Klotz, "Spectrophotometric investigation of the interactions of proteins with organic anions", J. Amer. Chem. Soc, vol. 68, pp. 2299-2304, November 1946.

[2] I.M. Klotz, R. "Burkhard and J. Urkuhart, "Structural specificities in the interactions of some organic ions with serum albumin”, J. Amer. Chem. Soc., vol. 74, pp. 202-208, 1952.

[3] G.E. Dobretsov, B. Polyak, N. Smolina, T. Babushkina, T. Syrejshchikova, T. Klimova, V. Sverbil, A. Peregudov, Yu. Gryzunov, and O. Sarkisov, "Interaction of a fluorescent probe, CAPIDAN, with human serum albumin", J. Photochem. Photobiol. A: Chemistry, vol. 251, pp. 134-140, January 2013.

[4] Yu.A. Gryzunov and G.E. Dobretsov, "Natural conformation of human serum albumin and its changes in pathology", in Protein Conformation: New Research, Ed. L.B. Roswell. Nova Publishers, New York, 2008, pp. 125-159.

[5] D.R. Sellers and G.A. Ghiron, "Role of the tryptophan fluorescent state in the ultraviolet-induced inactivation of beta-trypsin", Photochem. Photobiol., vol. 18, pp. 393-402, 1973.

[6] D.B. Calhoun, J.M. Vanderkooi, G.R. Holtorn, and nS.W.
Englander, "Protein fluorescence quenching by small molecules: protein penetration versus solvent exposure", Proteins: Structure, Function, and Genetics, vol. 1(2), pp. 109-115, October 1986.

[7] N.V. Smolina, Yu.A. Gryzunov, N.M. Maximova, G.E. Dobretsov, M.G. Uzbekov, E.Yu. Misionzhnik, and O.P. Vertogradova, "Characteristics of albumin molecule binding centers in patients with anxious depression. Study by the fluorescence quenching method", Bull. Exp. Biol. Med., vol. 144(5), pp. 674-676, November 2007 [Bull. Exp. Biol. Med., vol. 144(11), p. 514, 2007].

[8] T.I. Syrejshchikova, Yu.A. Gryzunov, N.V. Smolina, A.A. Komar, M.G. Uzbekov, E.Yu. Misionzhnik, and N.M. Maksimova, "Subnanosecond fluorescent spectroscopy of human serum albumin as a method for estimation of depression therapy efficiency", Laser Physics, vol. 20, pp. 1074-1078, November 2010.

[9] S.M. Andrade, S.M. Costa, J.W. Borst, A. van Hoek, and A.J. Visser, "Translational and rotational motions of albumin sensed by a non-covalent associated porphyrin under physiological and acidic conditions: a fluorescence correlation spectroscopy and time resolved anisotropy study", J. Fluoresc., vol. 18, pp. 601-610, May-July 2008.

[10] M. Banerjee, U. Pal, A. Subudhhi, A. Chakrabarti, and S. Basu, "Interaction of Merocyanine 540 with serum albumins: photophysical and binding studie", J. Photochem. Photobiol. B, vol. 108, pp. 23-33, March 2012.

[11] P. Bolel, N. Mahapatra, and M. Halder, "Optical spectroscopic exploration of binding of Cochineal Red A with two homologous serum albumins", J. Agric. Food. Chem., vol. 60, pp. 3727-3734, April 2012.

[12] O. Stern and M. Volmer, „Über die Abklingzeit der Fluoreszenz", Physikalische Zeitschrift, vol. 20, pp. 183-188, 1919.

[13] J.R. Lakowicz, Principles of Fluorescence Spectroscopy, Springer, 2006, p. 256.

[14] G.E. Dobretsov, T. I. Syreishchikova, Yu. A. Gryzunov, N. V. Smolina, and A. A. Komar, "Features of the binding of the fluorescent probe K-35 to albumin", Biophysics , vol. 55, , pp. 182-187, March-April 2010.

[15] G.E. Dobretsov, T.I. Syreishchikova, and N.V. Smolina, "Molecular mobility of a fluorescent probe in binding sites of an albumin molecules", Biophysics, vol. 56, pp. 403-406, May-June 2011.

[16] U. Kragh-Hansen, V.T.G. Chang, and M. Otagiri, "Practical aspects of the ligand-binding and enzymatic properties of human serum albumin", Biol. Pharm. Bull., vol. 25, pp. 695-704, June 2002.

[17] J. Ghuman, P.A. Zunszain, I. Petitpas, A.A. Bhattacharya, M. Otagiri, and S.Curry, "Structural basis of the drug-binding specificity of human serum albumin", J. Mol. Biol., vol. 353, pp. 38-52, October 2005.

[18] V.T. Chuang and M. Otagiri, "Stereoselective binding of human serum albumin", Chilarity, vol. 18(93), pp. 159-166, February 2006.

[19] Handbook of Chemistry and Physics, Ed. D.R. Line, CRC Press, 2001.

[20] T. Shimizu, „Chemical components of firework composi- 
tions", in: K. Kosanke, B. Kosanke, I. von Maltitz, T. Shimizu, M. A. Wilson, N. Kubota, C. Jennings-White, D. Chapman, Pyrotechnic Chemistry, 2004, p.30.

[21] M.A. Kiselev, Yu.A. Gryzunov, G.E. Dobretsov, and M.N. Komarova, "The size of human serum albumin molecules in solution”, Biophysics, vol. 46, pp. 402-405, 2001.

[22] E.L. Gelamo, R. Itri, A. Alonso, J.V. da Silva, and M. Tabak, "Small-angle X-ray scattering and electron paramagnetic resonance study of the interaction of bovine serum albumin with ionic surfactants", J. Colloid Interface Sci., vol. 277, pp. 471- 482, September 2004.

[23] Watanabe H., Kragh-Hansen U., Tanase S., Nakajou K., Mitarai M., Iwao Y., Maruyama T., and Otagiri M. "Conformational stability and warfarin-binding properties of human serum albumin studied by recombinant mutants", Biochem. J., vol. 357 (Pt 1), pp. 269-274, July 2001. 